\title{
Quelle place pour la formation à l'environnement des futur.e.s enseignant.e.s en adaptation scolaire et sociale?
}

Carine Villemagne et Enrique Correa Molina

\section{(2) OpenEdition}

\section{Journals}

Édition électronique

URL : https://journals.openedition.org/ere/7399

DOI : $10.4000 /$ ere.7399

ISSN : 2561-2271

Éditeur

Centr'ERE

Référence électronique

Carine Villemagne et Enrique Correa Molina, « Quelle place pour la formation à l'environnement des futur.e.s enseignant.e.s en adaptation scolaire et sociale? », Éducation relative à l'environnement [En ligne], Volume 16 - 1 | 2021, mis en ligne le 10 mars 2021, consulté le 16 juin 2021. URL : http:// journals.openedition.org/ere/7399; DOI : https://doi.org/10.4000/ere.7399 


\title{
Quelle place pour la formation à l'environnement des futur.e.s enseignant.e.s en adaptation scolaire et sociale?
}

\author{
Carine Villemagne et Enrique Correa Molina
}

Dans cet article, nous nous intéressons aux possibilités d'intégration de l'éducation relative à l'environnement (ERE) au sein d'un programme spécifique de formation des futur.e.s enseignant.e.s soit celui du Baccalauréat en adaptation scolaire et sociale (BASS) de l'Université de Sherbrooke où les deux auteurs exercent leur pratique enseignante et déploient une partie de leurs recherches. Un tel baccalauréat est centré sur l'enseignement du français et des mathématiques à des élèves ayant des troubles d'apprentissage, des difficultés d'adaptation scolaire et sociale ainsi que des handicaps et de la déficience légère ou moyenne aux cycles du primaire et secondaire. Les contenus couverts par les plans d'activité pédagogique du BASS vont généralement de la première année du primaire à la deuxième année du secondaire. Ainsi que Berryman (2006-2007, p. 147) le mentionnait, «Les didactiques des matières de base instrumentales dominent dans les curriculums » et c'est encore plus vrai dans un baccalauréat spécialisé comme le nôtre puisque les crédits de formation sont majoritairement consacrés à la didactique du français et des mathématiques (Université de Sherbrooke, 2021). Nous nous intéressons au cas particulier de ce baccalauréat en réalisant une recherche diagnostique portant sur les dimensions de formation actuellement reliées à l'ERE au sein du BASS tout en mettant en évidence d'autres possibilités d'intégration. En effet, il s'agit du seul programme de formation initiale à l'enseignement de la Faculté d'éducation qui ne possède aucun cours portant explicitement sur l'ERE. Ainsi après avoir situé les référents conceptuels qui constituent notre cadre d'analyse, nous explorons les problématiques contextuelles qui se retrouvent en toile de fond de notre travail de recherche à savoir, comment l'ERE ou les questions d'environnement sont traitées au sein des programmes de formation de l'école québécoise et au sein du récent référentiel de la profession enseignante? 
2 Nous ferons ensuite part des objectifs de notre recherche diagnostique et de la méthodologie déployée pour brosser un état de la situation de l'ERE au sein du programme de BASS. Les résultats montrent, comme nous le verrons, une intégration somme toute embryonnaire, mais aussi des avenues intéressantes d'enrichissement qui sont à ce jour non exploitées. Ces résultats alimenteront ultérieurement les propositions d'amélioration pour l'intégration de l'ERE au sein du programme de BASS, qu'elles soient constitutives du programme ou à la marge de celui-ci.

\section{Repères théoriques : l'éducation relative à l'environnement}

3 L'éducation relative à l'environnement est un champ de recherche et de pratique déployant une riche diversité de propositions au Québec comme à l'échelle internationale. D'emblée, l'approche critique que nous adoptons constitue le filtre à travers lequel nous analyserons les programmes de formation de l'école québécoise destinés aux élèves du primaire et du secondaire, les programmes universitaires visant la formation à l'enseignement des futur.e.s enseignant.e.s, particulièrement celui du BASS et le référentiel de compétences guidant l'organisation de ce programme. En effet, la formation des futur.e.s enseignant.e.s influence fortement la mise en œuvre des programmes de formation de l'école québécoise et ainsi la réussite scolaire des élèves. Aussi, nous adoptons la définition selon laquelle l'éducation relative à l'environnement a pour objet la relation à l'environnement, ici celles des jeunes élèves comme celles des étudiant.e.s universitaires, dans une visée d'harmonisation du réseau de relations personnes-société-environnement (Sauvé, 1997). Une telle ERE intègre trois perspectives qui teintent la manière d'aborder cette dimension éducative en problématisant différemment les enjeux d'apprentissage (Sauvé, 1997, p. 19-23) : la perspective environnementale "qui concernela dégradation de l'environnement ", est axée sur la résolution de problèmes d'environnement; la perspective éducationnelle "qui concernel'aliénation des personnes et des groupes sociaux au regard de leur milieu de vie ", est centrée sur le développement des personnes et de leur groupe d'appartenance; la perspective pédagogique cherche quant à elle, à améliorer les conditions d'apprentissage et d'enseignement.

4 S'intéressant à la nature structurelle, politique, éthique et idéologique des questions environnementales, l'ERE serait un processus d'apprentissage tout au long de la vie au cours duquel les personnes tissent un rapport sensible et affectif à leur environnement, examinent les fondements des crises socioécologiques qui les affectent, développent leur conscience socioécologique et leur désir d'engagement, mobilisent leurs expériences ainsi que leurs savoirs dans l'exercice d'une praxis créative et collective contribuant à l'amélioration de la qualité de leur environnement ainsi que l'ensemble des écosystèmes (Villemagne, Agundez-Rodriguez, Daniel et Beaudoin, 2018). L'éducation relative à l'environnement s'actualise dans un savoir-agir qui mobilise en contexte, des savoirs, des savoir-faire et des savoir-être. L'ERE participerait donc à la construction d'une écocitoyenneté. En ce qui concerne cette dernière, Séguin, de Coninck et Tremblay (2005, p. 19) constate qu'il s'agit d'un néologisme qui interroge la citoyenneté, l'environnement, les interrelations entre les deux et qui sous-tend l'adoption d'une vision globale et systémique. Pour Sauvé (2013, p. 21), l'écocitoyenneté se devrait d'être «critique, compétente, créative, engagée, capable et désireuse de 
participer aux débats publics, à la recherche de solutions et à l'innovation écosociale ». L'écocitoyenneté représente une intégration des dimensions environnementales dans les différentes facettes de la vie quotidienne de la population en général non en vue d'exercer une « responsabilité civique » de l'homme envers son milieu (Roesch, 2003, In Tremblay, 2005, p. 27), mais en vue d'exercer une responsabilité environnementale envers l'ensemble du vivant. Sauvé (2000) propose même la notion de responsabilité intégrale qui selon elle "amène à clarifier les liens étroits (mais rarement précisés) entre responsabilitê, conscience, luciditê, réflexivitê, libertê, autonomie, authenticité, engagement, courage, solidaritê et sollicitude ».

Aussi, Naoufal (2016, p. 19) reprend les trois différents types d'écocitoyens suggérés par Westheimer et Kahne (2004) où le type " écocitoyen critique et participant » serait le moins fréquemment rencontré alors qu'il serait le plus abouti. Un tel écocitoyen :

«S'investit dans sa communauté, tout en déconstruisant les causes des problématiques qu'il s'efforce de résoudre. Cuvrant pour l'avènement de la justice environnementale, cette forme d'écocitoyenneté est fondée sur des valeurs de justice, de coopération, de criticité, de participation, de vivre-ensemble, de solidarité, de diversité, d'interdépendance et de résistance créative. Ancré dans la praxis, un programme éducatif approprié au développement de cette écocitoyenneté conjugue la pédagogie de projet, la pédagogie critique, l'éducation expérientielle ainsi que des stratégies d'éducation relative aux valeurs environnementales, tels le débat, le jeu de rôle et l'analyse de controverses socioécologiques ".

Dans le contexte de la recherche qui est le nôtre, ce sont à la fois les élèves du primaire et $\mathrm{du}$ secondaire (généralement en classe d'adaptation scolaire et sociale) qui sont ciblés par le développement d'une telle écocitoyenneté, mais aussi les étudiant.e.s universitaires de baccalauréat qui enseigneront à ces élèves. L'absence de formation de ces derniers en ERE entraine le fait que peu d'initiatives scolaires visent l'intégration de l'ERE au sein des disciplines enseignées. Nous essaierons de mettre en évidence les éléments actuels de formation reliés à l'ERE au sein des programmes ainsi que certaines possibilités à valoriser puisque, comme nous le montrerons ci-après, les programmes de l'école québécoise comportent des occasions de formation à l'environnement.

\section{Les programmes de formation en jeu}

7 Examinons d'abord le Programme de Formation de l'École Québécoise (PFEQ, 2006 a et 2006 b) au regard de l'éducation relative à l'environnement

$8 \mathrm{Au}$ Québec, les questions environnementales sont abordées dans le cadre du Programme de Formation de l'École québécoise (PFEQ, 2006a ; 2006b) à travers les domaines généraux de formation ainsi que certains domaines disciplinaires tels l'univers social, la science et la technologie ainsi que l'éthique et la culture religieuse.

9 Tout d'abord, un domaine général de formation correspond « un ensemble de grandes questions que les jeunes doivent affronter. Il rapproche les savoirs disciplinaires des préoccupations quotidiennes de l'élève et lui donne plus de prise sur la réalité » ( $\mathrm{PFEQ}$, 2006a, p. 42). Deux domaines généraux de formation semblent inspirants pour l'ERE et pourraient constituer des points possibles d'ancrage pour une éducation relative l'environnement. Il s'agit d'abord du domaine "Environnement et consommation » dont l'intention éducative est « [ $\mathrm{d}$ »] amener l'élève à entretenir un rapport dynamique avec son milieu, tout en gardant une distance critique à l'égard de l'exploitation de 
l'environnement, du développement technologique et des biens de consommation" (PFEQ, 2006a, p. 47). Remarquons que le rapport au monde qui sous-tend cette intentionnalité serait ainsi celui d'un environnement, réservoir de ressources. Ce sont donc des ressources à exploiter dans une perspective consumérisme. Il s'agit ensuite du domaine général "Vivre ensemble et citoyenneté » dont l'intention éducative est de " permettre à l'élève de participer à la vie démocratique de l'école ou de la classe et de développer des attitudes d'ouverture sur le monde et de respect de la diversité (PFEQ, 2 006a, p. 50); dans son intentionnalité, le rapport à l'environnement, n'apparait pas comme une dominante, mais pourrait y trouver quand même une «niche" de développement.

Si le caractère transversal des domaines généraux de formation pouvait paraitre prometteur, puisqu'ils devraient constituer des lieux de convergences et d'intégration de différentes disciplines, dans les faits, il apparaît que l'enseignement des disciplines demeure cloisonné. Par ailleurs, ne faisant l'objet d'aucune évaluation ministérielle des apprentissages, les domaines généraux de formation semblent avoir été de grands oubliés tant dans la formation des futur.e.s enseignant.e.s que dans l'éducation des jeunes élèves.

11 Quant aux domaines dits disciplinaires, une rapide analyse des programmes aux cycles du primaire et au premier cycle du secondaire met en évidence le rôle potentiel des domaines de l'Univers social, de la Science et la technologie et de l'Éthique et la culture religieuse, au regard du déploiement de l'ERE. Un bref regard sur des dimensions abordées dans ces domaines donne un premier aperçu des possibilités.

En ce qui concerne le domaine d'apprentissage de l'Univers social au primaire, la compétence 1 Lire l'organisation d'une société sur son territoire vise à contribuer à « la prise de conscience des liens qui existent entre la satisfaction des besoins, l'utilisation des ressources et l'aménagement du territoire contribu[ant] (...) à la responsabilisation de l'élève face à l'environnement considéré comme un bien collectif» (PFEQ, 2006a, p. 172). De plus, la compétence 2 Interpréter le changement dans une société et sur son territoire, vise à contribuer au « développement d'attitudes et de valeurs essentielles à l'exercice du rôle de citoyen » (PFEQ, 2006a, p. 174).

13 Au premier cycle du secondaire (PFEQ, 2006b, p. 296), le domaine de l'Univers social serait étroitement lié aux deux domaines généraux de formation décrits ci-haut, les disciplines de la géographie et de l'histoire et l'éducation à la citoyenneté permettant à l'élève de «comprendre les réalités sociales pour assumer ses responsabilités de citoyen » (p. 298). La compétence 3 en géographie Construire sa conscience citoyenne à l'échelle planétaire invite l'élève à examiner les actions humaines de la manière suivante (PFEQ, 2006b, p. 312) :

«[L'élève] s'efforce de repérer [les actions humaines] qui favorisent une gestion responsable et une utilisation rationnelle des ressources. Il reconnait les actions humaines qui s'avèrent économiquement équitables, respectueuses de l'environnement, justes sur le plan social et adapté à la culture des sociétés qui occupent les territoires. (...) il apprend à devenir citoyen du monde ».

14 Quant à l'Histoire et l'éducation à la citoyenneté au premier cycle du secondaire (PFEQ, 2006b), les compétences de ce domaine sont orientées vers la construction d'une conscience citoyenne sociétale et interrelationnelle.

Pour ce qui est du domaine des Sciences et technologie, aux cycles du primaire (PFEQ, 2006a, p. 143), il est mentionné que la science et la technologie sont importantes pour 
« comprendre le monde dans lequel nous vivons et pour s'y adapter » et pour prendre " conscience de l'évolution du rapport que l'homme entretient avec la nature à travers les âges... »; le vivant y est abordé dans ses usages pour la consommation humaine (p. 149).

16 Au premier cycle du secondaire (PFEQ, 2006b), les compétences ciblées en Science et technologie sont les mêmes qu'au primaire et l'élève doit désormais «saisir la complexité des relations que les êtres vivants entretiennent avec leur milieu » (p. 267). La question de l'interdépendance des systèmes à l'échelle planétaire dans le domaine de l'environnement est relevée. La gestion des déchets, la réduction des émissions de polluants, la protection de la faune et de la flore, l'amincissement de la couche d'ozone ou encore les enjeux éthiques des biotechnologies (etc.) sont abordés dans une perspective de responsabilisation et de développement durable (p. 270). La démarche d'enseignement-apprentissage de résolution de problème est très présente et les contenus de formation reliés à «l'univers du vivant» abordent les traités internationaux sur la protection de l'environnement (p.285). Les deux domaines généraux de formation sont explicitement arrimés à ce domaine disciplinaire (p. 275). Enfin, examinons le domaine de l'Éthique et culture religieuse. Aux cycles du primaire (PECR, 2008) ainsi qu'au premier cycle du secondaire, le domaine du développement personnel comprend le programme Éthique et culture religieuse dont les compétences $1 /$ réfléchir sur des questions éthiques, 2/ pratiquer le dialogue et 3/ manifester une compréhension du phénomène religieux suivent une progression d'un cycle d'études à un autre et dont les liens avec le domaine général de formation «Environnement et consommation » sont formulés ainsi (p. 283) :

«En discutant de la relation d'interdépendance entre les êtres humains et les autres êtres vivants, on peut amener les élèves, par exemple, à examiner l'impact de leurs habitudes de consommation sur l'environnement. De même, en s'intéressant à des groupes ou à des personnes témoignant d'un sens des responsabilités à l'égard des êtres vivants, les élèves pourront mieux saisir le lien étroit qui unit l'être humain et son environnement $"$.

18 Quant au domaine général «Vivre ensemble et citoyenneté », le programme d'Éthique et culture religieuse y entretient des liens privilégiés compte tenu de son intention éducative, telle que nous l'avons mentionnée plus haut.

Les mots-clés qui reviennent dans ces trois domaines ou sous-domaines sont ceux de l'interdépendance des personnes et des écosystèmes, de la responsabilisation face à l'environnement, de la gestion des ressources juste, équitable et respectueuse de l'environnement afin d'assurer un développement durable. Ce qui transparait dans notre courte analyse est le potentiel intéressant pour l'ERE des démarches d'enseignement-apprentissage et des compétences transversales qui sont mobilisées comme celle de l'exercice d'un jugement critique. Néanmoins la principale limite qui traverse l'ensemble du PFEQ (2006a ; 2006b) et du PECR (2008) est l'inscription de ces derniers dans une perspective de développement durable qui, selon la conception adoptée, ne remet pas en cause le paradigme socioculturel de type industriel-rationnel qui organise notamment la société québécoise (Bertrand et Valois, 1999). Les programmes adoptent ce cadre de référence, conservant les perspectives dominantes de l'environnement dans la société actuelle, à savoir celles de l'environnementressource et puis de l'environnement-problème, dont les solutions émergent de la science et secondairement, du dialogue. Enfin, quelle que soit la discipline, l'ERE n'est jamais explicitement nommée. 

l'éducation relative à l'environnement. Au Québec, cette formation a reposé jusqu'en 2020 sur le document gouvernemental intitulé «La formation à l'enseignement. Les orientations. Les compétences professionnelles » (MEQ, Raymond et Gauthier, 2001). Ce dernier, qui présentait un référentiel de douze compétences professionnelles, servait de balise à l'élaboration de tout programme visant la formation à l'enseignement. Dans le cadre de la consultation 2017-2020 ayant trait à la refonte du référentiel de la profession enseignante, Sauvé et coll. (2020) mettaient judicieusement en lumière que l'ancien référentiel de compétences ne faisait aucunement mention»des enjeux environnementaux et du vivre ensemble " qui sont particulièrement préoccupants à l'heure actuelle. C'est aussi au regard de ce constat, que la Coalition Éducation Environnement et Écocitoyenneté ${ }^{1}$ appuyait la proposition d'une Stratégie québécoise d'éducation en matière d'environnement et d'écocitoyenneté (Sauvé, L., Asselin, H. et coll., 2019) destinée tant aux milieux d'enseignement formels que non formels, pour les motifs suivants :

«[...] tous les indicateurs de la santé de la planète sont au rouge [...] les mesures prises depuis 25 ans n'ont pas donné les résultats escomptés; [...] les problèmes environnementaux sont avant tout des problèmes anthropiques; [...]) les réponses à apporter concernent nos modes de vie [...] l'éducation est l'outil de transformation sociale le plus puissant que nous ayons.

L'ajout d'une nouvelle compétence professionnelle au référentiel en place était alors suggéré par Lucie Sauvé et coll. (2020) qui la décrivait ainsi « situer ses interventions pédagogiques et les apprentissages des élèves au regard des réalités écologique et sociale du milieu pour favoriser la construction d'une vision critique du monde actuel et à venir, et le développement d'une identité et d'un pouvoir-agir écocitoyens ». Soutenu par une analyse bibliographique approfondie, le Centr'ERE (2017) mettait par ailleurs en lumière les résultats des recherches portant sur les effets bénéfiques de l'intégration d'une éducation relative à l'environnement auprès des jeunes, favorisant leur réussite en tant qu'élèves, mais aussi en tant que personnes intégrales et citoyen.ne.s de ce monde. Son apport aux élèves ayant des besoins particuliers comme ceux que les enseignant.e.s en adaptation scolaire et sociale encadrent, y était aussi souligné (Centr'ERE, 2017, p. 3).

Or malgré les représentations politiques et stratégiques effectuées par la Coalition Éducation Environnement et Écocitoyenneté, le nouveau référentiel en vigueur pour la profession enseignante (ME, 2020) n'intègre pas cette nouvelle compétence suggérée. En effet, le nouveau référentiel comporte treize compétences professionnelles exposées dans le tableau 1. 
Tableau 1 : Les 13 compétences du référentiel (2020)

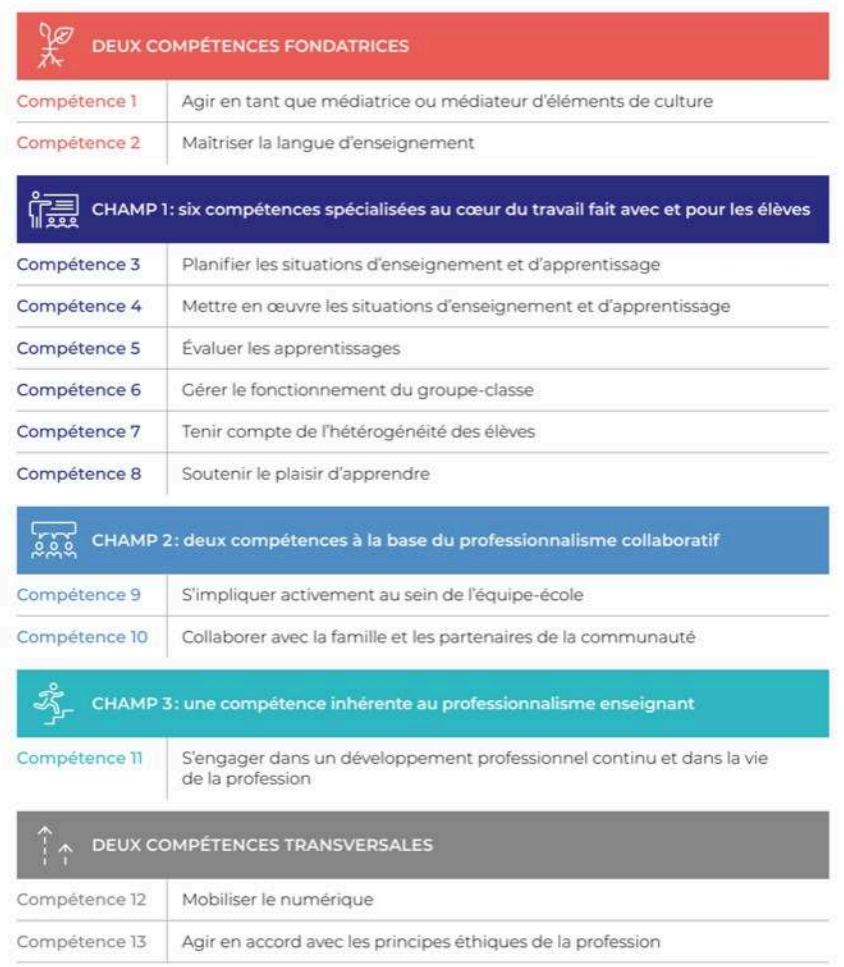

Dans ce document ministériel, il est au mieux fait mention que (ME, 2020, p. 14) : « depuis la publication du précédent référentiel, les attentes sociales à l'égard de l'école et de la profession enseignante se sont multipliées, tandis que l'environnement écologique, social, culturel et économique dans lequel naissent, grandissent et évoluent les nouvelles générations s'est complexifié (Fullan, 2020).»

Nous devons donc «faire avec » ce nouveau référentiel de la profession enseignante (2020) et nous avons relevé que la compétence 1 (CP1) dudit référentiel "agir en tant que médiatrice ou médiateur d'éléments de culture » laissait entrevoir des possibilités d'intégration. En effet, dans une perspective sociologique rapportée par Legendre (2005) :

[La culture] est : expression de la vie sociale; ensemble des phénomènes sociaux (religieux, moraux, scientifiques, techniques, etc.) propres à une communauté, à une société humaine ou à une civilisation; ensemble de manières de voir, sentir, percevoir, penser, s'exprimer, réagir... ensemble des modes de vie, des traditions, des normes, des mœurs, des valeurs, des aspirations qui distingue les membres d'une collectivité et qui cimente son unité à une époque. [...] La culture c'est d'abord l'ouverture à des valeurs universelles (Tenzer,1989).

Or selon la perspective que nous adoptons de l'éducation relative l'environnement, le rapport à l'environnement est un rapport culturel qui se doit de prendre de plus en plus de place dans nos vies afin d'enrayer la crise socioécologique majeure à laquelle nos sociétés font face. Le PFEQ (2006a, 2006b) ainsi que le PECR (2008) insistent aussi sur l'importance de la construction du rapport au monde des élèves. Ce qui diffère en revanche, entre notre vision et celles des programmes gouvernementaux, ce sont les éléments constitutifs et les visées de ces programmes. 


\section{Objectifs et méthodologie de la recherche diagnostique}

Avant de mettre en évidence des éléments d'intégration de l'ERE, ou de questions environnementales au sein du programme de BASS, nous explicitons nos objectifs de recherche ainsi que la méthodologie de recherche que nous avons adoptée.

Nos objectifs sont les suivants :

- Décrire et analyser des modalités d'intégration de l'ERE dans le programme de formation initiale à l'enseignement, le BASS de l'Université de Sherbrooke

- Formuler de pistes d'amélioration de l'intégration de l'ERE au sein de ce programme, notamment en matière de formation à l'ERE.

Pour atteindre ces objectifs, nous avons eu recours à la méthodologie de l'étude de cas : il s'agissait en effet de rendre compte des caractéristiques et de la complexité de «systèmes limités » (Smith, 1978, In Merriam, 1998, p. 19). Dans cette recherche, l'étude de cas simple favorise une description holistique et riche du cas qu'est le programme de BASS. La collecte de données a fait appel à des stratégies diversifiées soit l'analyse documentaire (Boisvert, 2004) des plans d'activités pédagogiques ciblés pour leur pertinence au regard des objectifs poursuivis (Univers social, Science et technologie, Éthique et culture religieuse), un groupe de discussion (Geoffrion, 2004) avec deux chargés de cours qui enseignent les plans d'activités pédagogiques ciblés, une entrevue avec un dernier chargé de cours non disponible lors du groupe de discussion ainsi qu'un sondage réalisé auprès des étudiant.e.s de $4^{\mathrm{e}}$ année du BASS qui ont validé tous les cours se rapportant aux domaines de l'Univers social, de la Science et technologie ainsi que l'Éthique la culture religieuse. Les résultats de ce sondage ne sont pas présentés dans cet article faute de place et feront l'objet d'un autre article.

\section{L'analyse des plans d'activités pédagogiques}

Les résultats de l'analyse de l'intégration d'une éducation en matière d'environnement et d'écocitoyenneté au sein du BASS auxquels les auteurs sont rattachés, sont exposés. Ils permettent de mettre en évidence l'intégration ou non de l'ERE dans la formation initiale des futur(e)s enseignant.e.s en identifiant des «niches potentielles de déploiement ».

L'analyse des six plans d'activités pédagogiques (deux par domaine disciplinaire) tend d'abord à montrer tant au primaire qu'au secondaire, que les plans demeurent les mêmes, et ce pour les trois domaines analysés. L'organisation structurelle des plans d'activités pédagogiques étant homogène, nous avons pu analyser ceux-ci selon les cibles de formation, les compétences visées au regard du référentiel de compétences, les démarches d'enseignement-apprentissage, les contenus de formation et les lectures suggérées. Rappelons que les domaines ciblés (reliés aux programmes d'enseignement) sont la didactique de l'Univers social (tableau 2), la didactique de la Science et de la technologie (tableau 3) ainsi que l'Éthique et la culture religieuse (tableau 4).

31 L'analyse des plans d'activités pédagogiques de la didactique de l'Univers social (tableau 2) met en évidence plusieurs possibilités d'arrimage pour l'ERE plutôt que des propositions existantes. Tout d'abord la CP1 est ciblée par les plans de cours. Ensuite, il apparaît que l'éducation à la citoyenneté est à l'étude au moyen notamment de 
lectures. De plus, des thématiques abordées en géographique et des stratégies d'apprentissage pourraient être facilement mobilisées pour intégrer l'ERE, comme le montrent les éléments surlignés en gras dans le tableau 2.

Tableau 2 : Didactique de l'Univers social, primaire et secondaire et ERE

\begin{tabular}{|c|c|}
\hline Élément d'analyse & Résultats d'analyse (existant et potentialités) reliés à l'ERE (en gras) \\
\hline Cible de formation & $\begin{array}{l}\text { S'initier à l'enseignement des sciences humaines au primaire et au } \\
\text { secondaire auprès d'élèves handicapés et en difficulté d'adaptation ou } \\
\text { d'apprentissage et s'approprier les contenus de géographie et } \\
\text { d'éducation à la citoyenneté. Maitriser les démarches et les outils } \\
\text { privilégiés pour enseigner l'Univers social. }\end{array}$ \\
\hline Compétences ciblées & $\begin{array}{l}\text { CP1 « Agir en tant que professionnel(le) héritier... », CP2 } \\
\text { «Communiquer clairement... », CP3 « Concevoir de situations } \\
\text { d'enseignement-apprentissage (SEA)....» ainsi que CP5, CP7, CP8 et } \\
\text { CP11 }\end{array}$ \\
\hline $\begin{array}{l}\text { Démarches } \\
\text { d'enseignement- } \\
\text { apprentissage }\end{array}$ & $\begin{array}{l}\text { Conceptions de SEA en contexte d'adaptation scolaire et sociale. } \\
\text { Stratégie d'enseignement : Analyse critique. Ateliers. Discussion et } \\
\text { débats }^{2} \text {. }\end{array}$ \\
\hline $\begin{array}{l}\text { Contenus des séances } \\
\text { d'apprentissage }\end{array}$ & $\begin{array}{l}\text { Fondements épistémologiques des programmes du primaire et du } \\
\text { secondaire. } \\
\text { Thématique : «Territoires à l'étude et les enjeux géographiques »- } \\
\text { Exploration de conceptions d'élèves relativement aux sciences } \\
\text { humaines (géographie, histoire et éducation à la citoyenneté). } \\
\text { Conception de situation d'enseignement-apprentissage. Exploitation } \\
\text { pédagogique de diverses sources d'information. Analyse critique } \\
\text { d'outils didactiques variés. }\end{array}$ \\
\hline Lectures suggérées & 8 textes intégrant l'éducation à la citoyenneté \\
\hline
\end{tabular}

L'analyse des plans d'activités pédagogiques de la didactique de la Science et de la technologie (tableau 3) met en évidence qu'une des cibles explicites de formation est de prendre conscience des enjeux sociaux, culturels, environnementaux et éthiques contemporains associés. Les domaines généraux de formation sont étudiés et certaines activités pédagogiques comme la visite du Musée de la nature et des sciences de Sherbrooke laissent croire que le rapport à la nature et aux sciences ainsi que certaines questions environnementales vives sont étudiés.

Tableau 3 : Didactique de la Science et de la technologie, primaire et secondaire et ERE

\begin{tabular}{|l|l|}
\hline Élément d'analyse & $\begin{array}{l}\text { Résultats d'analyse (existant et potentialités) reliée à l'ERE (en } \\
\text { gras) }\end{array}$ \\
\hline Cible de formation & $\begin{array}{l}\text { S'approprier les programmes québécois au primaire et au } \\
\text { secondaire / prendre conscience des enjeux sociaux, culturels, } \\
\text { environnementaux et éthiques contemporains associés. } \\
\text { Apprentissage de différents types de savoirs (connaissances, } \\
\text { savoir-faire-habiletés et savoir-être-attitudes). }\end{array}$ \\
\hline Compétences ciblées & $\begin{array}{l}\text { CP1 « Agir en tant que professionnel(le) héritier... » et CP3 } \\
\text { "Concevoir de situations d'enseignement-apprentissage....» }\end{array}$ \\
\hline $\begin{array}{l}\text { Démarches d'enseignement- } \\
\text { apprentissage }\end{array}$ & $\begin{array}{l}\text { Réseau conceptuel, modélisation, expérimentation, } \\
\text { apprentissage par projet, résolution de problème, conférences, } \\
\text { visite de musée, enseignement magistral, ateliers et discussion. }\end{array}$ \\
\hline $\begin{array}{l}\text { Contenus des séances } \\
\text { d'apprentissage }\end{array}$ & $\begin{array}{l}\text { Contenus du programme et didactique des sciences. Musée de la } \\
\text { Nature et des Sciences. Interdisciplinarité ET sciences et } \\
\text { technologies. }\end{array}$ \\
\hline Lectures suggérées & $\begin{array}{l}\text { Bégin (2006) Finalités de l'enseignement des sciences et } \\
\text { domaines généraux de formation... }\end{array}$ \\
\hline
\end{tabular}

L'analyse des plans d'activités pédagogiques de la didactique de l'Éthique et culture religieuse (tableau 4) met en évidence plusieurs possibilités d'arrimage pour l'ERE plutôt que des propositions existantes. Tout d'abord la CP1 est ciblée par les plans de 
cours. Par ailleurs, plusieurs stratégies pédagogiques mobilisées seraient très pertinentes pour favoriser le développement de la pensée critique en ERE.

Tableau 4 : Éthique et culture religieuse, primaire et secondaire et ERE

\begin{tabular}{|l|l|}
\hline Élément d'analyse & $\begin{array}{l}\text { Résultats d'analyse (existant et potentialités) reliés à l'ERE } \\
\text { (en gras) }\end{array}$ \\
\hline Cible de formation & $\begin{array}{l}\text { Se familiariser avec les principes et les contenus du } \\
\text { programme d'éthique et culture religieuse au moyen de } \\
\text { démarches pédagogiques expérimentées lors des cours }\end{array}$ \\
\hline Compétences ciblées & $\begin{array}{l}\text { CP1 « Agir en tant que professionnel(le) héritier... », } \\
\text { CP3 « Concevoir des situations d'enseignement- } \\
\text { apprentissage... », CP11 « S'engager dans une démarche } \\
\text { individuelle et collective de développement professionnel » } \\
\text { et CPI23 « Agir de façon éthique et responsable dans } \\
\text { l'exercice de ses fonctions ». }\end{array}$ \\
\hline $\begin{array}{l}\text { Démarches d'enseignement- } \\
\text { apprentissage }\end{array}$ & $\begin{array}{l}\text { Prise de notes, exploration de ses propres conceptions, } \\
\text { discussion de groupe, développement de situation } \\
\text { d'enseignement-apprentissage, formation au débat et au } \\
\text { dialogue, le dilemme éthique, la communauté de } \\
\text { recherche philosophique. }\end{array}$ \\
\hline Contenus d'apprentissage & $\begin{array}{l}\text { Contenus du programme d'éthique et culture religieuse et } \\
\text { démarches pédagogiques. }\end{array}$ \\
\hline Lectures suggérées & $\begin{array}{l}\text { Textes à lire qui n'indiquent pas de lien direct ou indirect } \\
\text { avec 1'ERE }\end{array}$ \\
\hline
\end{tabular}
lors de la discussion, et ce au regard des points de vue des chargée.e.s de cours qui les mettent en œuvre. Ces points de vue sont exposés dès à présent selon le même ordre de présentation que celui des plans d'activités pédagogiques.

\section{Le point de vue de chargé.e.s de cours}

Un canevas de groupe de rencontre a été élaboré pour discuter avec les chargé.e.s de cours (CC) en entrevue individuelle (1 personne) ou en groupe (2 personnes). Comprenant 14 questions, ce canevas interrogeait les préoccupations environnementales des CC, leurs visions de l'environnement ainsi que l'intégration actuelle ou potentielle (à quelles conditions ?) des questions d'environnement et de l'ERE lors de leur enseignement auprès des étudiant.e.s du BASS (Annexe).

Les CC interviewé(e)s sont deux femmes et un homme et les trois ont un baccalauréat en enseignement dans un domaine autre que l'adaptation scolaire et sociale. Deux détiennent une maitrise alors que la troisième détient un postdoctorat. Seulement une des trois CC enseigne exactement dans sa discipline de formation initiale alors que les deux autres ont une expertise développée au cours de leur trajectoire professionnelle. Tous les trois ont environ 20 années d'expériences professionnelles depuis l'obtention de leur baccalauréat. Pour l'un des trois, l'expérience n'est pas toujours ancrée en enseignement. En tant que CC, les répondant(e)s cumulent entre 9 et 16 ans d'enseignement à l'université. Il et elles considèrent être responsables d'activités pédagogiques jugées peu importantes par leurs étudiant.e.s d'adaptation scolaire et sociale et ont d'ailleurs peu de crédits à enseigner. Sur cent-vingt crédits du baccalauréat, chacun(e) enseigne 4 crédits ( 60 heures) par domaine (pour chaque domaine, ce sont deux cours de deux crédits chacun, l'un pour le profil des futur.e.s enseignant.e.s au primaire et l'autre pour le profil des futur.e.s enseignant.e.s au 
secondaire). Ce sont donc 12 crédits sur 120 qui sont attribués aux trois domaines disciplinaires que nous avons ciblés pour leur potentiel d'intégration de l'ERE.

La chargée de cours en Univers social ( $\mathrm{CC} 1$ ) se déclare préoccupée par les injustices sociales et écologiques depuis longtemps. Le respect de l'environnement fait partie de ses choix de vie et elle tente d'adopter des «habitudes de vie » (CC1) cohérentes avec ses valeurs. Pour $\mathrm{CC} 1$, poser des actions concrètes est fondamental. Pour cette personne, l'environnement est rattaché à l'identité de l'individu qui fait partie de l'environnement, celui du quotidien et est composé d'un ensemble de systèmes interreliés. Les dimensions politique et sociale de l'environnement sont importantes pour elle.

Quand il est question d'enseigner les disciplines qui se rattachent à l'Univers social (géographie, histoire et éducation à la citoyenneté), CC1 note que selon les cycles d'enseignement, les questions d'environnement peuvent être intégrées différemment en importance. Par exemple au premier cycle du secondaire, la géographie aborde de grands enjeux sociaux, territoriaux et environnementaux. Au primaire, les questions d'environnement et d'injustice sont plus abordées dans les rapports nord / sud et dans l'analyse de certains faits historiques; l'environnement d'aujourd'hui est un héritage du passé. Éduquer à l'environnement pourrait donc avoir sa place, même si ce n'est pas une compétence prescrite. En effet, le développement de valeurs et de comportements favorables à l'environnement a sa place auprès des futur.e.s enseignant.e.s. Ces derniers doivent avoir développé ces valeurs et comportements, doivent être conscients et critiques pour qu'ils puissent à leur tour former leurs élèves. Toutefois, selon la CC1, si ses étudiant.e.s semblent bien informé.e.s des questions environnementales (à l'aide d'informations parfois tirées de sources peu fiables), elle constate que leur agir ne semble pas cohérent avec leur niveau d'information.

Notons que $\mathrm{CC} 1$ possède une solide expérience de ce qu'elle nomme «des projets thématiques» ( $\mathrm{CC} 1)$ au sein desquels elle intégrait différentes disciplines pour encourager la motivation de ses élèves en difficulté. Elle a aussi une maitrise où le "débat » (CC1) en tant que stratégie pédagogique fait partie de son cadre de référence. Cette personne l'utilise dans son enseignement, car elle le juge comme étant un puissant moyen d'animation en poussant ses étudiant.e.s à réfléchir et à agir en cohérence avec leurs choix. Elle interroge leur identité, dimension de leur être et dimension de leur appartenance à un tout plus vaste. Pour $\mathrm{CC} 1$, intégrer les questions d'environnement en ce moment est très à propos compte tenu de l'actualité et des grands défis socioécologiques auxquels la population fait face. Il y aurait lieu de les intégrer selon une approche programme et non seulement dans les disciplines dont elle est responsable, car ces dernières bénéficient de trop peu de crédits d'enseignement. Le manque de temps pour réaliser une telle intégration est ainsi souligné.

Quant à la chargée de cours en Science et technologie (CC2), celle-ci affirme avoir un intérêt pour les questions d'environnement. Pour elle, conserver l'environnement, ses ressources, ses matières premières et la nature vise à se garantir une meilleure vie. L'environnement, c'est aussi de «la chimie » (CC2) et c'est un ensemble de systèmes imbriqués de l'échelle microscopique à macroscopique. L'environnement possède des liens avec son champ d'expertise qu'est la didactique des sciences : elle cherche à faire " comprendre le monde et les phénomènes qui nous entourent pour mieux agir " (CC2). Elle reconnait que son champ d'expertise constitue un creuset fertile pour intégrer l'ERE puisque le domaine « Environnement et consommation » est inscrit dans 
le Programme de Formation de l'École Québécoise (cycles primaire et secondaire). Toutefois, l'ERE devrait être envisagée non de façon ponctuelle, mais sur le long terme. Cette chargée de cours constate une incohérence entre le discours en faveur de l'environnement de ses étudiant.e.s de baccalauréat et leurs actes alors qu'ils ont bénéficié d'une réforme qui intégrait sous diverses formes les questions d'environnement. Quant à l'intégration de l'ERE dans ses activités pédagogiques, la problématisation de concepts scientifiques est souvent une occasion de parler de questions d'environnement. Mais elle souligne que tous les cours du BASS devraient être concernés étant donné le caractère interdisciplinaire de l'environnement et l'ampleur des changements à faire au niveau de la société. Être un passeur culturel c'est « comprendre pour bien agir et faire des choix judicieux en tant que citoyen » (CC2). La question des valeurs à choisir est fondamentale, mais ce sont surtout les savoirs d'ordre cognitifs sur lesquels elle insiste le plus avec ses étudiant.e.s. Les démarches pédagogiques qu'elle enseigne à ses étudiant.e.s ainsi que la présentation d'exemples concrets (conférence d'une enseignante, trousse pédagogique, visite au Musée de la Nature et des Sciences de Sherbrooke) tendent à leur montrer comment les sciences peuvent être facilement convoquées en classe.

41 Pour finir, cette chargée de cours croit qu'il faudrait qu'on lui explicite mieux les liens entre environnement et éducation et elle devrait se former à l'ERE. Tout comme la CC1, elle pense que l'intégration de l'ERE est une problématique propre à l'ensemble du programme de BASS : « ce n'est pas d'un spécialiste dont nous avons besoin »; puis elle ajoute « $\mathrm{j}$ 'aimerais mener une réflexion sur ce qu'on peut faire, comme formatrice dans une classe avec des étudiant.e.s, comment je peux faire de l'ERE ; ce n'est pas clair dans ma tête " (CC2). En science et technologie, le peu de crédits d'enseignement dont elle dispose lui fait penser qu'elle manquerait de temps pour réaliser une véritable intégration. Elle souhaiterait avoir à disposition des ressources pédagogiques.

Le chargé de cours en Éthique et culture religieuse (CC3) considère avoir « une conscience environnementale extrêmement forte depuis le secondaire 1 » (CC3). Se déclarant « sensible » aux différentes problématiques environnementales existantes, il adopte des petits gestes au quotidien, car «chaque petit geste compte». Pour lui, l'environnement est associé à la nature qui entoure l'école, au milieu de vie des élèves où l'on peut y déployer des projets, à la biosphère, mais aussi aux problèmes écologiques.

43 Son mandat en tant que CC est de développer la pensée critique des futur.e.s enseignant.e.s. Ce ne sont pas les contenus des programmes ciblés qui comptent, mais l'apprentissage des processus d'enseignement-apprentissage qu'ils pourront ultérieurement mobiliser dans leurs classes. Les questions d'environnement ne sont pas traitées par ce $\mathrm{CC} 3$, mais il arrive que des étudiant.e.s choisissent de traiter de ces questions dans leur travail de session.

Selon lui, une intégration serait possible en utilisant une question d'environnement comme "prétexte» au service des processus d'enseignement-apprentissage qu'il fait expérimenter à ses étudiant.e.s. Par exemple, en proposant un dilemme éthique. Car son but premier est de développer la réflexion et la capacité d'argumentation, d'écoute et d'empathie chez ses étudiant.e.s. Il souhaite que ses étudiant.e.s enseignent différemment le programme "Éthique et culture religieuse " soit sortir des cahiers d'apprentissage conçus par les maisons d'édition pour la dimension éthique et de la présentation magistrale des différentes religions. En matière d'environnement, il ne 
souhaiterait pas adopter une posture cherchant à convaincre ses étudiant.e.s du bienfondé de changer d'attitudes et de comportements. Le manque de temps pour réaliser une telle intégration est aussi souligné.

\section{Discussion des résultats} est question d'environnement; ce qui n'est pas un mal en soi. S'ils reconnaissent tous les trois l'existence d'une crise aux multiples facettes et de divers problèmes environnementaux, l'une parle d'engagement et de justice sociale et environnementale, la suivante s'intéresse à conserver l'environnement et le dernier dit avoir une conscience environnementale très développée depuis sa jeunesse. Pour deux d'entre eux (un homme et une femme) cela les amène à poser des gestes au quotidien et pour les deux femmes chargées de cours, leur rôle éducatif est de faire que leurs étudiant.e.s du BASS passent de la parole consciente aux actes, en cohérence avec leur discours favorable à l'environnement. Pour les chargé.e.s de cours, l'environnement n'est pas perçu en extériorité à soi. On en fait partie, car l'environnement est un ensemble de systèmes interreliés; il est le milieu de vie des futur.e.s enseignant.e.s et de leurs élèves et il peut donner lieu à des projets collectifs.

Pour deux des domaines d'apprentissage, Univers social puis Éthique et culture religieuse, l'analyse des plans d'activités pédagogiques met en évidence que les domaines généraux de formation "Environnement et consommation" ainsi que «Vivre ensemble et citoyenneté » ne sont pas ciblés explicitement alors que le domaine général « Environnement et consommation » l'est en Science et technologie, comme en témoigne un "Texte à lire » recommandé dans un plan de cours. Cette situation est confirmée lors des rencontres avec les chargé.e.s de cours qui ne parlent pourtant pas des domaines généraux de formation comme porte d'entrée pour les questions d'ERE et d'éducation écocitoyenne.

En matière d'ERE, les trois chargé.e.s de cours ont du mal à nommer à quoi correspond ce champ et comment s'y prendre pour « faire de l'ERE » dans leurs cours. Pourtant les suggestions qu'ils évoquent sont fort intéressantes. Par exemple, les chargées de cours en Univers social et en Science et technologies souhaiteraient que l'ERE fasse partie l'approche programme du BASS. Rappelons qu'une telle approche induit une cohérence interne à la formation en «favorisant une meilleure intégration chez l'étudiant.e des acquis de formation et en soutenant la progression dans le développement des compétences attendues au terme de celle-ci. » (Legendre et David, 2012, p. 46).

Les objets d'apprentissage en matière d'environnement ne sont pas les mêmes selon les chargé.e.s de cours. Pour deux d'entre eux, il faut adopter de nouvelles valeurs (dimension morale) et des processus de pensée comme le jugement critique (dimension de la critique sociale); pour la dernière, il faut développer des savoirs pertinents (dimension cognitive) pour mieux intervenir (dimensions résolutique et pragmatique). Il est intéressant de noter que tous les éléments d'une compétence à développer ne se retrouvent pas dans le discours des chargé.e.s de cours, en particulier les savoir-faire et le savoir agir en contexte comme manifestation d'une compétence. Parmi les perspectives de l'ERE mises en lumière par Sauvé (1997), ce sont les perspectives environnementales et pédagogiques (l'environnement est un prétexte à l'apprentissage) qui sont présentes dans le discours des personnes interviewées.

Éducation relative à l'environnement, Volume 16 - 1 | 2021 
49 Du point de vue des démarches pédagogiques mises en valeurs dans les plans d'activités pédagogiques, il est possible de constater le grand potentiel d'intégration de l'ERE en lien avec l'apprentissage des questions environnementales vives. Le débat, la résolution de problème, le développement de projets intégrateurs, la communauté de recherche philosophique et bien sûr, la conception de situation d'enseignement-apprentissage dans chaque domaine disciplinaire montrent tant la diversité des approches utilisées que leur transférabilité pour l'ERE. Ces approches empruntées à la didactique générale ou au domaine de la formation morale sont fort pertinentes. Signalons ici toutefois cette limite : la dimension concrète et expérientielle de l'apprentissage (sortir hors les murs de la classe) n'est abordée qu'indirectement par des exemples lors de conférences ou à travers des ressources pédagogiques existantes.

50 La formation des chargé.e.s de cours à l'éducation relative à l'environnement est suggérée comme une avenue d'appropriation pour ces formateurs et formatrices qui expriment le souhait de mieux saisir le champ notionnel qui s'y rattache ainsi que les approches d'intégration en collaboration avec les personnes responsables des autres disciplines enseignées au BASS, notamment le français et les mathématiques. Il n'est pas donc question de créer un cours spécifique sur l'ERE, qui d'ailleurs, comme le fait remarquer judicieusement une chargée de cours, n'est pas une compétence professionnelle prescrite dans le cadre du référentiel de compétences de la profession enseignante.

\section{Conclusion}

51 Les résultats de la recherche que nous avons menée rendent compte des dimensions de formation actuellement reliées à l'ERE au sein du BASS offert à l'Université de Sherbrooke. Elles sont très peu élaborées. Ces résultats mettent donc surtout en évidence diverses possibilités d'intégration et de développement pour l'ERE. Selon notre étude, les chargé.e.s de cours les plus directement concerné.e.s par l'intégration de l'ERE au BASS, et ce, en raison des disciplines enseignées, sont globalement favorables à une telle intégration au programme BASS, mais non uniquement au sein de leurs cours. Il serait donc très pertinent d'élargir le débat auprès de l'ensemble des chargé.e.s de cours et des professeur.e.s qui enseignent au BASS afin de mieux saisir les possibilités réelles quant à l'intégration de l'ERE. L'enjeu est de taille au regard des jeunes qui sont en classes d'adaptation scolaire et sociale. Comme le souligne le Centr'ERE (2017, p. 4), « tous les types d'élèves ont besoin de contextes d'apprentissage stimulants et signifiants, en particulier ceux qui éprouvent des difficultés. (...) Des recherches mettent en lumière les bienfaits du temps passé dehors et en nature par exemple pour améliorer l'attention des élèves ».

52 Toutefois, ne faisant pas l'objet d'une compétence prescrite par le ministère de l'Éducation dans le nouveau référentiel de compétences professionnelles (ME, 2020), l'intégration de l'ERE continue de dépendre soit des milieux scolaires et de leurs équipes-écoles, soit des équipes universitaires responsables des baccalauréats en enseignement, comme celui du BASS. D'autres cadres nous apparaissent influencer aussi un tel développement, en particulier les cadres généraux universitaires qui déterminent la gestion de l'Université de Sherbrooke. L'éducation au développement durable pourrait ainsi entrer en concurrence avec l'ERE, en raison de l'existence d'activités pédagogiques en ce sens et surtout d'une politique universitaire de 
développement durable (2500-017) (Université de Sherbrooke, 2005). Plus récemment, l'Université de Sherbrooke se questionne sur le type de diplômé qu'elle souhaite valoriser auprès des futurs étudiant.e.s et des employeurs en vue de leur intégration au «marché du travail». Le projet de faire des étudiant.e.s «des diplômé(e)s écoresponsables" a été exploré lors d'un forum universitaire (Vice-rectorat au développement durable et à l'administration, 2019). Toutes ces propositions devront dès lors faire l'objet d'un arrimage et plus largement d'une mise en cohérence au regard des finalités poursuivies.

\section{BIBLIOGRAPHIE}

Bertrand Y. et Valois, P. (1999). Fondements éducatifs pour une nouvelle société. Montréal : Éditions Nouvelles.

Berryman, T. (2006-2007). Des pistes pour institutionnaliser l'éducation relative à l'environnement dans la trajectoire obligatoire de la formation initiale à l'enseignement primaire. Éducation relative à l'environnement : Regards - Recherches - Réflexions, 6, 137-158.

Boisvert, D. (2004). La recherche documentaire et informationnelle, Dans Gauthier, B. (dir), Recherche sociale : de la problématique à la collecte de données. 4e édition (p. 85-102). Sainte-Foy : Presses de l'Université du Québec.

Centr'ERE - Centre de recherche sur l'éducation et la formation relatives à l'environnement et à l'écocitoyenneté (2017). Mémoire pour une politique de la réussite éducative présenté par le Centre de recherche en éducation et formation relatives à l'environnement et à l'écocitoyenneté. Éducation, environnement, écocitoyenneté : une combinaison féconde pour la réussite éducative. Montréal : Université du Québec à Montréal. Consulté sur https://centrere.uqam.ca/wpcontent/uploads/2017/03/Memoire-_CentrERE_UQAM-Reussite_Educative-2016.pdf.

Geoffrion, P. (2016). Le groupe de discussion, Dans Gauthier, B. et Bourgeois, I. (dir), Recherche sociale : de la problématique à la collecte de données. 6e édition (p. 401-1425). Sainte-Foy : Presses de l'Université du Québec.

Legendre, M. et David, R. (2012). Les référentiels de compétences : dans quelle mesure contribuent-ils à assurer une plus grande cohérence de la formation ? Dans Desjardins J. (éd.), La formation des enseignants en quête de cohérence (p. 43-65). Louvain-la-Neuve, Belgique : De Boeck Supérieur.

Legendre, R. (2005). Dictionnaire actuel de l'éducation, 4 e édition. Montréal : Éditions Guérin Eska.

ME - ministère de l'Éducation (2020). Référentiel de compétences professionnelles. Profession enseignante. Québec : Gouvernement du Québec, ministère de l'Éducation. Consulté sur https:// cdn-contenu.quebec.ca/cdn-contenu/adm/min/education/publications-adm/devenirenseignant/referentiel_competences_professionnelles_profession_enseignante.pdf ?1606848024

MELS - ministère de l'Éducation, du Loisir et du Sport (2006a). Programme de formation de l'école québécoise. Cycles du Primaire. Québec : ministère de l'Éducation du Loisir et du Sport, 
MELS - ministère de l'Éducation, du Loisir et du Sport (2006b). Programme de formation de l'école québécoise. Premier cycle du secondaire. Québec : ministère de l'Éducation du Loisir et du Sport.

MELS - ministère de l'Éducation, du Loisir et du Sport (2008). Programme d'éthique et culture religieuse. Québec : ministère de l'Éducation du Loisir et du Sport.

Merriam, S. (1998). Qualitative research and case study applications in education. San Francisco : Jossey-Bass.

MEQ, Raymond, D. et Gauthier, C. (2001). La formation à l'enseignement. Les orientations. Les compétences professionnelles. Québec : gouvernement du Québec, ministère de l'Éducation.

Naoufal, N. (2016). Connexions entre la justice environnementale, l'écologisme populaire et l'écocitoyenneté. Vertigo - La revue électronique en sciences de l'environnement, 16 (1). Consulté sur https://id.erudit.org/iderudit/1037574ar

Potvin, M., Borri-Anadon, C. et Larochelle-Audet, J. (2015). Rapport sur la prise en compte de la diversité ethnoculturelle, religieuse et linguistique dans les orientations et compétences professionnelles en formation à l'enseignement. Montréal : Observatoire sur la formation à la diversité et l'équité.

Sauvé, L. (1997). Pour une éducation relative à l'environnement. Montréal : Guérin, $2^{\mathrm{e}}$ édition.

Sauvé, L. (1998). L'éthique de la responsabilitẻ en éducation relative à l'environnement, en préparation.

Sauvé, L. (2000). L'éducation relative à l'environnement entre modernité et postmodernité. Les propositions du développement durable et de l'avenir viable. Dans A. Jarnet, Jickling, B., L. Sauvé, Arjen Wals et Priscilla Clarkin (dir.), The Future of Environmental Education in a Postmodern World ? (p57-71). Whitehorse : Canadian Journal of Environmental Education.

Sauvé, L., Asselin, H. et coll. (2019). Stratégie québécoise d'éducation en matière d'environnement et d'écocitoyenneté - Défis, visions et pistes d'action. Une recherche-développement partenariale (2014-2019). Montréal/UQAM : Centre de recherche en éducation et formation relatives à l'environnement et à l'écocitoyenneté. Consulté sur https://www.coalition-educationenvironnement-ecocitoyennete.org/wp-content/uploads/2019/07/Strategie-Editioncomplete.pdf

Sauvé, L. et coll. (2020) Une analyse de la proposition de Référentiel de compétences professionnelles pour la profession enseignante et sa formation. Dans le cadre de la Consultation menée par la Direction de la valorisation et de la formation du personnel enseignant du ministère de l'Éducation et de l'Enseignement supérieur du Québec. Centre de recherche en éducation et formation relatives à l'environnement et à l'écocitoyenneté - Centr'ERE - UQAM. Consulté sur https:// centrere.uqam.ca/contributions-aux-debats-publics/le-centrere-contribue-a-la-revision-dureferentiel-de-competences-professionnelles-en-enseignement/

Séguin, M., P. De Coninck et F. Tremblay, 2005, Présentation du dossier : Le contexte planétaire de l'écocitoyenneté, Nouvelles Pratiques Sociales, 18(1), p. 18-25.

Université de Sherbrooke (2021). Baccalauréat en adaptation scolaire et sociale. Sherbrooke :

Université de Sherbrooke, Département d'études sur l'adaptation scolaire et sociale. Consulté sur https://www.usherbrooke.ca/admission/programme-pdf/246/baccalaureat-en-adaptationscolaire-et-sociale/

Université de Sherbrooke (2005). Politique de développement durable 2500-017. Sherbrooke : Conseil d'administration, Université de Sherbrooke. 
Villemagne, C., Agundez-Rodriguez, A., Beaudoin, C., Daniel, J. et Sauvé, L (2018). L'éducation relative à l'environnement des adultes dans une perspective radicale, Rapport de recherche.

Sherbrooke : Éditions du Centr'ERE

\section{ANNEXES}

\section{Questions à l'intention des chargé.e.s de cours}

- Pouvez-vous me décrire les cours de votre domaine d'expertise que vous enseignez au BASS ? Est-ce votre formation initiale qui vous a conduit à les enseigner ou d'autres motivations éventuelles?

- De manière générale, les questions d'environnement qui sont abordées dans l'espace sociopolitique vous intéressent-elles ? Lesquelles en particulier?

- Comment qualifiez-vous votre rôle sur les questions d'environnement en tant que passeur culturel au regard de la compétence 1 que les étudiant.e.s ont à développer au BASS «agir en tant que professionnel(le) héritier critique et interprète d'objets de savoirs et de culture dans l'exercice de ses fonctions »?

- Les questions d'environnement sont-elles abordées dans vos plans d'activités pédagogiques (sciences et environnement, éthique et environnement, univers social et environnement) au regard des contenus d'apprentissage disciplinaires ou des domaines généraux de formation (Environnement et consommation/vivre ensemble et citoyenneté) ? Si non pourquoi ? Si oui quelles sont-elles ?

- Quels objectifs poursuivez-vous auprès de vos étudiant.e.s quand vous intégrez les questions d'environnement à votre enseignement?

- Quelles stratégies d'enseignement-apprentissage utilisez-vous sur ces questions d'environnement avec vos étudiant.e.s (y compris les lectures) ?

- Dans ce cadre-ci, quelles compétences du référentiel de compétences du BASS visez-vous?

- Avez-vous dû acquérir des connaissances ou développer des compétences spécifiques en lien avec l'environnement, et l'éducation à l'environnement pour enseigner vos cours au BASS ? De quelles manières?

- Quelle est la réceptivité de vos étudiant.e.s lorsque vous abordez les questions d'environnement au regard de vos disciplines d'enseignement?

- La question du cheminement primaire ou secondaire introduit-elle des différences dans la façon d'enseigner les questions reliées à l'environnement?

- Dans les activés d'enseignement-apprentissage que vous réalisez dans vos disciplines respectives, les aspects social, culturel, économique et politique des problématiques environnementales ? Précisez et de quelle manière ?

- Parmi les objets d'apprentissage suivant quels sont ceux que vous abordez dans vos plans d'activités pédagogiques? Praxis environnementale; attitudes et sentiments qui suscitent un agir favorable à l'environnement; valeurs morales et éthique de l'environnement; savoirs d'ordre cognitif contribuant à l'alphabétisation environnementale; habiletés de résolution de problèmes environnementaux; comportements; attitudes et valeurs conformes à une croyance ou à une philosophie religieuse ou spirituelle.

- Quelles sont les représentations de l'environnement mises de l'avant dans le cadre de votre enseignement? 
- Quelles sont les représentations de la relation éducation-environnement mises de l'avant dans le cadre de votre enseignement?

\section{NOTES}

1. www.coalition-education-environnement-ecocitoyennete.org/

\section{RÉSUMÉS}

Les auteurs ont réalisé une recherche diagnostique visant à mettre en évidence dans quelle mesure l'éducation relative à l'environnement (ERE) fait l'objet d'apprentissages au sein du programme de formation initiale à l'enseignement où ils interviennent, soit le Baccalauréat en adaptation scolaire et sociale (BASS) de l'Université de Sherbrooke. Ils ont aussi exploré les possibilités de son intégration. Le BASS a ainsi constitué le cas à l'étude dans un contexte où seul ce Baccalauréat de la Faculté d'éducation ne propose aucune offre de cours explicite de formation concernant l'ERE. Pour ce faire, l'analyse des plans d'activités pédagogiques reliés aux domaines disciplinaires de l'Univers social, de la Science et technologie et de l'Éthique et culture religieuse a été réalisée ; un groupe de discussion et une entrevue ont également été menés auprès des trois chargé.e.s de cours qui interviennent dans ces domaines. Les résultats de cette étude ont permis de mettre en évidence des éléments de contenus de même que des démarches pédagogiques présentant des liens avec l'approche des questions environnementales chez les futur.e.s enseignant.e.s. Les préoccupations environnementales des chargé.e.s de cours ainsi que leurs représentations de l'environnement montrent leur ouverture à une intégration future de l'ERE au sein de leurs domaines disciplinaires comme au sein du BASS selon une approche programme.

The authors carried out diagnostic research aimed at highlighting the extent to which environmental education (EE) was the subject of learning within the initial training program in which they teach, namely the bachelor's program in special education (or BASS, for Baccalauréat en adaptation scolaire et sociale) at the Université de Sherbrooke.. They also explored the possibilities of its integration. The BASS was thus their case study in a context where only this bachelor's program offered by the Faculty of Education does not propose any explicit course on EE. To do so, the analysis of educational activity plans related to the disciplinary areas of the Social Universe, Science and Technology and Ethics and Religious Culture was carried out; a focus group and an interview were also conducted with the three lecturers responsible for these educational activities. These analyzed data highlights to what extent content elements and educational approaches are presenting links with environmental issues and EE. The environmental concerns of the lecturers as well as their environmental conceptions show their openness to a future integration of EE within their disciplinary fields as well as within the BASS according to a globally approach of this program. 
INDEX

Keywords : environmental education, initial teacher training, skills framework

Mots-clés : éducation relative à l'environnement, formation initiale des enseignants, référentiel de compétences

\section{AUTEURS}

\section{CARINE VILLEMAGNE}

Carine Villemagne est professeure titulaire en éducation des adultes à la Faculté d'éducation de l'Université de Sherbrooke. Ses travaux de recherche portent sur les adultes faiblement scolarisés ainsi que l'éducation relative à l'environnement des adultes dans le cadre de pratiques d'alphabétisation. Elle travaille à la fois en contextes d'éducation formelle et non formelle dans la perspective d'enrichir et de nourrir leurs pratiques éducatives interdisciplinaires.

\section{ENRIQUE CORREA MOLINA}

Enrique Correa Molina est professeur titulaire à la Faculté d'éducation de l'Université de Sherbrooke. Il possède une importante expertise en formation initiale à l'enseignement puisqu'il a été responsable de la formation pratique durant de nombreuses années au sein du baccalauréat en adaptation scolaire et sociale de l'Université de Sherbrooke. 